Commun. math. Phys. 30, 229 - 247(1973)

(C) by Springer-Verlag 1973

\title{
The Ideal Boson Gas in an External Scalar Potential
}

\author{
E. B. Davies
}

Mathematical Institute, St. Giles, Oxford, U.K.

Received October 10, 1972

\begin{abstract}
We consider a new way of going to the infinite volume thermodynamic limit for a finite density quantum system and apply it to the case of an ideal Boson gas. We describe two procedures for calculating the particle density in the thermodynamic limit, one local and one global, and show that they give different values for the density. Further calculations show that this discrepancy is caused by lack of macroscopic translation invariance of the system, which is not apparent at the microscopic level. We calculate the limiting value of the expectation function of the Weyl operators both above and below the critical density for Bose-Einstein condensation, and show that the condensate has paradoxical properties of a similar type to those recently discovered for the rotating Boson gas.
\end{abstract}

\section{$\S$ 1. Formulation of the Problem}

We consider a system of spin-less particles, taking the single particle space to be $\mathscr{H}=L^{2}\left(\mathbb{R}^{3}\right)$. We suppose the particles are confined by an external scalar potential so that the single particle Hamiltonian is

$$
H_{L} f(x)=-\frac{1}{2} \Delta f(x)+V\left(L^{-1} x\right) f(x) .
$$

We take $V$ to be non-negative with

$$
\lim _{\|x\| \rightarrow \infty} V(x)=+\infty
$$

so that the Hamiltonians are semi-bounded with discrete spectrum. In the limit $L \rightarrow \infty$ the Hamiltonian converges at least formally to a translation invariant free Hamiltonian. We denote the eigenvalues of $H_{L}$ in increasing order by $\left\{E_{L, n}\right\}_{n=0}^{\infty}$ and the corresponding normalised eigenfunctions by $\left\{\phi_{L, n}\right\}_{n=0}^{\infty}$. We let $\mathscr{F}$ be the Boson Fock space over

$$
\mathscr{F}=\sum_{n=0}^{\infty} \bigotimes_{\text {sym }}^{n} \mathscr{H}
$$

and let $\sigma_{L, z}$ denote the trace class operator defined on $\mathscr{F}$ for $0<z<1$ and $\beta>0$ by

$$
\left\{\sigma_{L, z} \psi\right\}_{n}=\alpha_{L, z} z^{n} e^{n \beta E_{L, 0}} \bigotimes^{n} e^{-\beta H_{L}} \psi_{n}
$$


where $\psi_{n} \in \bigotimes_{\text {sym }}^{n} \mathscr{H}$ and $\alpha_{L . z}$ is chosen so that $\operatorname{tr}\left[\sigma_{L, z}\right]=1$; explicitly, [9, p. 199],

$$
\alpha_{L, z}=\prod_{n=0}^{\infty}\left(1-z e^{\left.\beta E_{L, 0}-\beta E_{L, n}\right)} .\right.
$$

This is the thermodynamic state for the fugacity $z$ and the inverse temperature $\beta[1,18]$. We shall keep $\beta$ constant throughout the paper, and therefore do not indicate the dependence of the various thermodynamic quantities on $\beta$.

We denote by $N$ the number operator on Fock space and define the global particle density function by

$$
\begin{aligned}
\varrho_{L, z} & =L^{-3} \operatorname{tr}\left[\sigma_{L, z} N\right] \\
& =L^{-3} \sum_{n=0}^{\infty} \sum_{m=1}^{\infty} z^{m} e^{\beta m E_{L, 0}-\beta m E_{L, n}} \\
& =L^{-3} \sum_{m=1}^{\infty} z^{m} e^{\beta m E_{L, 0}} \operatorname{tr}\left[e^{-\beta m H_{L}}\right]
\end{aligned}
$$

as may be seen by separate consideration of each mode $[1,9,12]$. There are also local particle number operators $N(E)$ defined for each Borel set $E \cong \mathbb{R}^{3}$ by

$$
N(E)=\int_{E} a^{*}(x) a(x) d^{3} x
$$

according to the conventional notation of quantum field theory. We define them rigorously by

$$
\begin{aligned}
\{N(E) \psi\}_{n}= & \left(P_{E} \otimes 1 \otimes \cdots \otimes 1\right) \psi_{n} \\
& +\left(1 \otimes P_{E} \otimes 1 \otimes \cdots \otimes 1\right) \psi_{n}+\cdots+\left(1 \otimes \cdots \otimes 1 \otimes P_{E}\right) \psi_{n}
\end{aligned}
$$

where $\psi_{n} \in \bigotimes_{\text {sym }}^{n} \mathscr{H}$ and $P_{E}$ is the projection in $\mathscr{H}$ given by

$$
\left(P_{E} \phi\right)(x)=\chi_{E}(x) \phi(x)
$$

for all $\phi \in \mathscr{H}$. It is fairly easy to show [1] that the number of particles in $E$

$$
\varrho_{L, z}(E)=\operatorname{tr}\left[\sigma_{L, z} N(E)\right]
$$

is given by

$$
\begin{aligned}
\varrho_{L, z}(E) & =\sum_{n=0}^{\infty} \sum_{m=1}^{\infty} z^{m} e^{\beta m E_{L, 0}-\beta m E_{L, n}}\left\|P_{E} \phi_{L, n}\right\|^{2} \\
& =\sum_{m=1}^{\infty} z^{m} e^{\beta m E_{L, 0}} \operatorname{tr}\left[e^{-\beta m H_{L}} P_{E}\right]
\end{aligned}
$$

which is a countably additive measure on $\mathbb{R}^{3}$. 
We take the thermodynamic limit by calculating the asymptotic forms of these expressions as $L \rightarrow \infty$. We define the global particle density

$$
\varrho_{z}=\lim _{L \rightarrow \infty} \varrho_{L, z}
$$

and the local particle density

$$
\varrho_{z}(a)=\lim _{L \rightarrow \infty} \varrho_{L, z}(\Omega+L a)
$$

where $a \in \mathbb{R}^{3}$ and $\Omega$ is any region of unit volume in $\mathbb{R}^{3}$. It turns out that the second limit is independent of $\Omega$ but not of $a$, and that $\varrho_{z} \neq \varrho_{z}(0)$. Section 2 is devoted to proving this fact and to finding its explanation in terms of the lack of macroscopic translation invariance of the system. In Section 3 we use the results to study the thermodynamic limit of the expectation functions of the Weyl operators, and in particular to study the Bose-Einstein condensate, which turns out to have rather singular behaviour.

An interesting case of the general theory is when $V$ is homogeneous of order $\alpha>0$, that is

$$
V(\lambda x)=\lambda^{\alpha} V(x)
$$

for all $x \in \mathbb{R}^{3}$ and all $\lambda \geqq 0$. It is then easy to show by change of scale that

and

$$
E_{L, n}=L^{-2 x /(x+2)} E_{1, n}
$$

The potential is determined by the region

$$
\Lambda=\left\{x \in \mathbb{R}^{3}: V(x)<1\right\}
$$

through the formula

$$
V(x)=\inf \left\{\lambda^{x}: \lambda^{-1} x \in \Lambda\right\} .
$$

The standard problem of the thermodynamic limit for an ideal Boson gas may be considered as the limiting case of a homogeneous potential of order $+\infty$, so that

$$
V(x)=\left\{\begin{array}{cll}
0 & \text { if } & x \in \Lambda \\
+\infty & \text { if } & x \notin \Lambda
\end{array} .\right.
$$

The relevant boundary condition is then $\phi=0$ on $\partial \Lambda$. This problem has been extensively studied $[1,9]$. It has also been shown, by the use of difficult estimates from the theory of partial differential equations, that the thermodynamic limit depends in an interesting manner not only on the shape of the region $A$ but also on the type of boundary conditions used $[12,17]$. 
We remark that for the potential

$$
V(x)=\omega^{2}\left(x_{1}^{2}+x_{2}^{2}+x_{3}^{2}\right) / 2
$$

which is homogeneous of order two, many of the estimates of the paper are unnecessary, since the eigenvalues and eigenfunctions of the Hamiltonian are well known [15], and $e^{-t H_{L}}$ may be written down explicitly as an integral operator [22].

We remark that several authors have discussed problems similar to ours for systems of classical particles $[7,8,14,16]$.

The author should like to thank J. T. Lewis and J. Pulè for providing him with preliminary manuscripts of their papers $[12,13]$, and the former particularly for outlining some results of his on the harmonic oscillator case of this theory.

\section{§ 2. Calculation of the Density Functions}

We start by defining precisely the class of potentials we shall consider. We suppose that $V$ is a continuous non-negative function on $\mathbb{R}^{3}$ such that

and

$$
\begin{aligned}
& \min \left\{V(x): x \in \mathbb{R}^{3}\right\}=0 \\
& \lim _{\|x\| \rightarrow \infty} V(x)\|x\|^{-\delta}=+\infty
\end{aligned}
$$

for some $\delta>0$. We define $H_{L}$ as the closure of the symmetric operator defined on the domain $C_{0}^{\infty}\left(\mathbb{R}^{3}\right)$ of $C^{\infty}$ functions of compact support in $\mathbb{R}^{3}$ by

$$
H_{L} f(x)=-\frac{1}{2} \Delta f(x)+V\left(L^{-1} x\right) f(x) .
$$

Proposition 2.1. $H_{L}$ is essentially self-adjoint on $C_{0}^{\infty}\left(\mathbb{R}^{3}\right)$ and is nonnegative with purely discrete spectrum. If its eigenvalues in increasing order, repeated according to multiplicity, are $\left\{E_{L, n}\right\}_{n=0}^{\infty}$ then

and

$$
0<E_{L, 0}<E_{L, 1} \leqq E_{L, 2} \ldots
$$

$$
\lim _{L \rightarrow \infty} E_{L, 0}=0 \text {. }
$$

The operator $e^{-t H_{L}}$ is of trace class on $\mathscr{H}$ for all $t>0$ and has a continuous non-negative integral kernel.

Proof. These are standard results from the theory of partial differential equations. A unified functional-analytical treatment well adapted to our present needs may be found in [6]. See also $[20,21]$.

We define the function $g_{\sigma}$ by

$$
g_{\sigma}(z)=\sum_{n=1}^{\infty} n^{-\sigma} z^{n}
$$

whenever this series converges [9]. 
Lemma 2.2. Let $V$ be homogeneous of order $\alpha>0$. Then for all $0<z<1$

$$
\lim _{L \rightarrow \infty} \varrho_{L, z}=(2 \pi)^{-3 / 2}|\Lambda| \Gamma(1+3 / \alpha) \beta^{-3(2+\alpha) / 2 \alpha} g_{3(2+x) / 2 \alpha}(z) .
$$

Proof. We use Eq. (1.15) together with an asymptotic estimate on the eigenvalues of $H_{1},[21$, p. 174], that as $\lambda \rightarrow \infty$

$$
\max \left\{n: E_{1, n} \leqq \lambda\right\} \sim \frac{\sqrt{2}}{3 \pi^{2}} \int_{\{x: V(x)<\lambda\}}\{\lambda-V(x)\}^{3 / 2} d^{3} x .
$$

We choose spherical coordinates and for any unit vector $u$ in $\mathbb{R}^{3}$ let $\phi(u)$ be the number determined by

$$
V\{\phi(u) u\}=1,
$$

that is $\phi(u)=V(u)^{-1 / x}$. Then if $A=\{x: V(x)<1\}$

while

$$
|\Lambda|=\int_{\|u\|=1} \int_{0 \leqq r \leqq \phi(u)} r^{2} d r d S(u)=\frac{1}{3} \int_{\|u\|=1} \phi(u)^{3} d S(u)
$$

$$
\begin{aligned}
\int_{V(x)<\lambda}\{\lambda-V(x)\}^{3 / 2} d^{3} x & =\int_{\|u\|=1} \int_{0 \leqq r \leqq \phi(u) \lambda^{1 / \alpha}}\left\{\lambda-r^{\alpha} \phi(u)^{-\alpha}\right\}^{3 / 2} r^{2} d r d S(u) \\
& =\lambda^{3 / 2} \int_{\|u\|=1}\{1-t\}^{3 / 2} \alpha^{-1} \lambda^{3 / \alpha} t^{3 / \alpha-1} \phi(u)^{3} d t d S(u) \\
& =3|\Lambda| \alpha^{-1} \lambda^{3 / 2+3 / \alpha} \Gamma(5 / 2) \Gamma(3 / \alpha) / \Gamma(5 / 2+3 / \alpha) .
\end{aligned}
$$

Therefore

where

$$
\max \left\{n: E_{1, n} \leqq \lambda\right\} \sim k_{\alpha} \lambda^{3 / 2+3 / x}=k_{\alpha} \lambda^{3(2+\alpha) / 2 \alpha}
$$

$$
k_{\alpha}=(2 \pi)^{-3 / 2}|\Lambda| \Gamma(1+3 / \alpha) / \Gamma(5 / 2+3 / \alpha) \text {. }
$$

Now letting

$$
\begin{aligned}
F_{L}(x) & =L^{-3} \max \left\{n: E_{L, n}-E_{L, 0} \leqq x\right\} \\
& =L^{-3} \max \left\{n: E_{1, n}-E_{1,0} \leqq L^{2 \alpha /(\alpha+2)} x\right\}
\end{aligned}
$$

we see that

$$
\lim _{L \rightarrow \infty} F_{L}(x)=k_{\alpha} x^{3(2+\alpha) / 2 \alpha}
$$

and also that for some $k$ independent of $L$ and $x$

Now

$$
0 \leqq F_{L}(x) \leqq k x^{3(2+\alpha) / 2 \alpha} .
$$

$$
\begin{aligned}
\varrho_{L, z} & =L^{-3} \sum_{n=0}^{\infty} z e^{\beta E_{L, 0}}\left(e^{\beta E_{L, n}}-z e^{\beta E_{L, 0}}\right)^{-1} \\
& =L^{-3} z(1-z)^{-1}+\int_{0}^{\infty} \beta z e^{\beta x}\left(e^{\beta x}-z\right)^{-2} F_{L}(x) d x .
\end{aligned}
$$


By the Lebesgue dominated convergence theorem we see that if $0<z<1$

$$
\begin{aligned}
\lim _{L \rightarrow \infty} \varrho_{L, z} & =\int_{0}^{\infty} \beta z e^{\beta x}\left(e^{\beta x}-z\right)^{-2} k_{x} x^{3(2+\alpha) / 2 \alpha} d x \\
& =\left[3 k_{\alpha}(2+\alpha) / 2 \alpha\right] \int_{0}^{\infty} z\left(e^{\beta x}-z\right)^{-1} x^{(6+\alpha) / 2 \alpha} d x \\
& =k_{\alpha} \Gamma(5 / 2+3 / \alpha) \beta^{-3(2+\alpha) / 2 x} g_{3(2+x) / 2 \alpha}(z) \\
& =(2 \pi)^{-3 / 2}|\Lambda| \Gamma(1+3 / \alpha) \beta^{-3(2+\alpha) / 2 \alpha} g_{3(2+\alpha) / 2 \alpha}(z)
\end{aligned}
$$

as required.

As a check we observe that as $\alpha \rightarrow \infty$ this converges to the standard expression for the particle density of an ideal Boson gas

$$
|\Lambda|(2 \pi \beta)^{-3 / 2} g_{3 / 2}(z) \text {. }
$$

However, the fact that the limit depends on the order of homogeneity of the potential even though the Hamiltonian is translation invariant in the limit $L \rightarrow \infty$, demands some explanation.

We may also calculate the limiting behaviour of the fugacity if the density is kept fixed, as in $[10,12]$. Given $\varrho>0$ we define $z(L)$ as the solution of

$$
\varrho_{L, z(L)}=\varrho \text {. }
$$

Lemma 2.3. Let $V$ be a potential which is homogeneous of degree $\alpha>0$ and let

$$
\varrho_{c}=(2 \pi)^{-3 / 2}|\Lambda| \Gamma(1+3 / \alpha) \beta^{-3(2+\alpha) / 2 \alpha} g_{3(2+\alpha) / 2 \alpha}(1) .
$$

Then if $0<\varrho<\varrho_{c}$ and $z(L)$ is defined by Eq. (2.18), $z(L)$ converges to $z$ where

$$
\varrho=(2 \pi)^{-3 / 2}|\Lambda| \Gamma(1+3 / \alpha) \beta^{-3(2+\alpha) / 2 \alpha} g_{3(2+\alpha) / 2 \alpha}(z) .
$$

On the other hand if $\varrho_{c} \leqq \varrho<\infty$ then $z(L)$ converges to 1 and the rate of convergence is given by

$$
\lim _{L \rightarrow \infty} L^{-3} z(L)\{1-z(L)\}^{-1}=\varrho-\varrho_{c} .
$$

Proof. From Equation 1.6 we see that $\varrho_{L, z}$ is a strictly monotonically increasing continuous function on $\{z: 0<z<1\}$ and that

$$
\lim _{z \downarrow 0} \varrho_{L, z}=0 ; \quad \lim _{z \uparrow 1} \varrho_{L, z}=+\infty .
$$

Therefore for every $\varrho>0$ there is a unique value of $z(L)$ satisfying Eq. (2.18). By Lemma 2.2 for each $0<z<1$ the function $\varrho_{L, z}$ converges to a limit $\varrho_{z}$ which is a continuous strictly monotonically increasing function on $\{z: 0 \leqq z \leqq 1\}$ with $\varrho_{1}=\varrho_{c}$. By well known properties of monotonic functions the convergence is uniform in each interval $\{z: \varepsilon \leqq z \leqq 1-\varepsilon\}$ 
and the Lemma quickly follows if $0<\varrho<\varrho_{c}$. It also follows by monotonicity that if $\varrho \geqq \varrho_{c}$ then $z(L)$ converges to 1 as $L \rightarrow \infty$. Now examining Equation 2.16 it is seen that the second term on the right hand side converges uniformly as $L \rightarrow \infty$ for all $0 \leqq z \leqq 1$. Therefore replacing $\varrho_{L . z}$ by $\varrho$ and $z$ by $z(L)$ and letting $L \rightarrow \infty$ we get

$$
\varrho=\lim _{L \rightarrow \infty} L^{-3} z(L)\{1-z(L)\}^{-1}+\varrho_{c}
$$

which completes the proof of the Lemma.

We leave the calculation of the global density function for a general potential until later on, and turn now to finding the asymptotic form of the local particle density function. We carry out this calculation for the general potential immediately.

Theorem 2.4. Let $V$ be a general potential. Then for each $a \in R^{3}$ and $0<z<1$

$$
\lim _{L \rightarrow \infty} \varrho_{L, z}(a)=(2 \pi \beta)^{-3 / 2} g_{3 / 2}\left(e^{-\beta V(a)} z\right) .
$$

Proof. We denote by $H_{0}$ the self-adjoint, non-negative operator which is the closure of $-\Delta / 2$ on the domain $C_{0}^{\infty}\left(\mathbb{R}^{3}\right)$. It is immediate that

$$
\lim _{L \rightarrow \infty}\left\|H_{L} \psi-\left\{H_{0}+V(0) 1\right\} \psi\right\|=0
$$

for all $\psi \in C_{0}^{\infty}\left(\mathbb{R}^{3}\right)$ so $H_{L}$ converges strongly to $\left(H_{0}+V(0) 1\right)$ in the generalised sense [11, p. 429]. This implies, $[5,11]$, that for all $t>0$

$$
S-\lim _{L \rightarrow \infty} e^{-t H_{L}}=e^{-t\left(H_{0}+V(0) 1\right)} .
$$

We let $P$ be the orthogonal projection of $L^{2}\left(\mathbb{R}^{3}\right)$ onto $L^{2}(\Omega)$ where $\Omega$ is any fixed bounded region of unit volume and note that $e^{-t H_{0}}$ is an integral operator with kernel

Since

$$
\begin{aligned}
k(t, x, y)=(2 \pi t)^{-3 / 2} & \exp \left[-\|x-y\|^{2} / 2 t\right] . \\
S-\lim _{L \rightarrow \infty} P e^{-t H_{L}} P & =P e^{-t H_{0}} P e^{-t V(0)} \\
\lim _{L \rightarrow \infty} \inf \operatorname{tr}\left[P e^{-t H_{L}}\right] & \geqq \operatorname{tr}\left[P e^{-t\left(H_{0}+V(0) 1\right)}\right] \\
& =e^{-t V(0)} \int_{\Omega} k(t, x, x) d^{3} x \\
& =(2 \pi t)^{-3 / 2} e^{-t V(0)} .
\end{aligned}
$$

Using the notation and results of [6] we note that since $V \gg 0$

$$
0 \ll e^{-t H_{L}} \ll e^{-t H_{0}}
$$

for all $t>0$ so

$$
0 \ll P e^{-t H_{L}} \ll P e^{-t H_{0}}
$$


and in consequence

$$
0 \leqq \operatorname{tr}\left[P e^{-t H_{L}}\right] \leqq(2 \pi t)^{-3 / 2} .
$$

We now prove that for all $t>0$

$$
\limsup _{L \rightarrow \infty} \operatorname{tr}\left[P e^{-t H_{L}}\right] \leqq(2 \pi t)^{-3 / 2} e^{-t V(0)} .
$$

Let $a<V(0)$ be any constant and let $\|x\|<c$ imply $V(x)>a$. Let $W_{L}$ be the bounded potential defined by

Then

$$
W_{L}(x)=\left\{\begin{array}{lll}
0 & \text { if } & \|x\|<L c \\
a & \text { if } & \|x\| \geqq L c
\end{array} .\right.
$$

$$
V\left(L^{-1} x\right) \geqq a-W_{L}(x)
$$

for all $x \in \mathbb{R}^{3}$, so

$$
0 \ll e^{-t H_{L}} \ll e^{-t\left(H_{0}+a-W_{L}\right)}
$$

for all $t>0$ and

$$
0 \leqq \operatorname{tr}\left[P e^{-t H_{L}}\right] \leqq \operatorname{tr}\left[P e^{-t\left(H_{0}+a-W_{L}\right)}\right] .
$$

The right hand side can be evaluated exactly, but to keep within the spirit of this work, we estimate it by functional analytical methods. Since $W_{L}$ is a bounded potential

$$
e^{-t\left(H_{0}+a-W_{L}\right)}=e^{-t\left(H_{0}+a\right)}+\int_{s=0}^{t} e^{-s\left(H_{0}+a\right)} W_{L} e^{-(t-s)\left(H_{0}+a-W_{L}\right)} d s
$$

by $[11$, p. 495$]$ so

Now

$$
\begin{aligned}
& \left\|P e^{-t\left(H_{0}+a-W_{L}\right)}\right\|_{\text {H.S. }} \leqq\left\|P e^{-t\left(H_{0}+a\right)}\right\|_{\text {H.S. }} \\
& \quad+\int_{s=0}^{t}\left\|P e^{-s\left(H_{0}+a\right)} W_{L}\right\|_{\text {H.S. }} d s .
\end{aligned}
$$

$$
\begin{aligned}
& \left\|P e^{-s\left(H_{0}+a\right)} W_{L}\right\|_{\mathrm{H} . S .}^{2} \\
& \quad=e^{-2 s a} a^{2}(2 \pi s)^{-3} \int_{x \in \Omega} \int_{\|y\|>L c} \exp \left[-\|x-y\|^{2} / s\right] d^{3} x d^{3} y
\end{aligned}
$$

which converges to zero as $L \rightarrow \infty$ uniformly in $s$ for $0 \leqq s \leqq t$. Therefore

$$
\lim _{L \rightarrow \infty}\left\|P e^{-t\left(H_{0}+a-W_{L}\right)}\right\|_{\text {H.S. }}=\left\|P e^{-t\left(H_{0}+a\right)}\right\|_{\text {H.S. }} .
$$

Now for any $K=K^{*} \geqq 0$

$$
\left\|P e^{-t K}\right\|_{\text {H.S. }}^{2}=\operatorname{tr}\left[P e^{-t K} e^{-t K} P\right]=\operatorname{tr}\left[P e^{-2 t K}\right]
$$


so Eq. (2.37) implies that

$$
\lim _{L \rightarrow \infty} \operatorname{tr}\left[P e^{-t\left(H_{0}+a-W_{L}\right)}\right]=\operatorname{tr}\left[P e^{-t\left(H_{0}+a\right)}\right]=e^{-t a}(2 \pi t)^{-3 / 2} .
$$

Combining this with Eq. (2.34) proves Eq. (2.30). Together with Eq. (2.27) we obtain

$$
\begin{aligned}
\lim _{L \rightarrow \infty} \operatorname{tr}\left[P e^{-t H_{L}}\right] & =\operatorname{tr}\left[P e^{-t\left(H_{0}+V(0)\right)}\right] \\
& =(2 \pi t)^{-3 / 2} e^{-t V(0)} .
\end{aligned}
$$

This, together with Eq. (2.25), implies by [3] that

$$
\lim _{L \rightarrow \infty}\left\|P e^{-t H_{L}} P-P e^{-t\left(H_{0}+V(0)\right)} P\right\|_{\mathrm{tr}}=0
$$

which implies, in particular, operator norm convergence.

We finally observe that

$$
\varrho_{L, z}(0)=\sum_{m=1}^{\infty} z^{m} e^{\beta m E_{L, 0}} \operatorname{tr}\left[P e^{-\beta m H_{L}}\right] .
$$

If $z<z_{1}<1$ then since $\lim _{L \rightarrow \infty} E_{L, 0}=0$, the series is dominated by the convergent series

$$
\sum_{m=1}^{\infty} z_{1}^{m} \operatorname{tr}\left[P e^{-\beta m H_{0}}\right]=\sum_{m=1}^{\infty} z_{1}^{m}(2 \pi m \beta)^{-3 / 2}
$$

and the individual terms in the series converge as we have already shown. Therefore

$$
\begin{aligned}
\lim _{L \rightarrow \infty} \varrho_{L, z}(0) & =\sum_{m=1}^{\infty} z^{m}(2 \pi m \beta)^{-3 / 2} e^{-m \beta V(0)} \\
& =(2 \pi \beta)^{-3 / 2} g_{3 / 2}\left(z e^{-\beta V(0)}\right)
\end{aligned}
$$

which concludes the proof of the Theorem in case $a=0$. The general result may be obtained by solving the same problem for the translated potential $V_{a}(x)=V(x+a)$.

We have now shown that $\varrho_{z}$ is not equal to $\varrho_{z}(0)$. The clue to the explanation of this lies in the fact that $\varrho_{z}(a)$ depends on $a$, suggesting that the mean particle density varies over distances which are very large compared with the thermal wavelength. For homogeneous potentials the situation is clarified by the following lemma.

Lemma 2.5. If $V$ is a homogeneous potential of order $\alpha>0$ and $0<z<1$ then

$$
\varrho_{z}=\int_{\mathbb{R}^{3}} \varrho_{z}(a) d^{3} a
$$


Proof. This is an explicit calculation carried out in polar coordinates.

$$
\begin{aligned}
\int_{\mathbb{R}^{3}} \varrho_{z}(a) d^{3} a & =\sum_{m=1}^{\infty}(2 \pi m \beta)^{-3 / 2} z^{m} \int_{\mathbb{R}^{3}} e^{-m \beta V(a)} d^{3} a \\
& =\sum_{m=1}^{\infty}(2 \pi m \beta)^{-3 / 2} z^{m} \int_{\|u\|=1}^{\infty} \int_{r=0}^{\infty} e^{-m \beta r^{\alpha} V(u)} r^{2} d r d S(u) \\
= & \sum_{m=1}^{\infty}(2 \pi m \beta)^{-3 / 2} z^{m} \int_{\|u\|=1}^{\infty} \int_{s=0}^{\infty} e^{-s}\{m \beta V(u)\}^{-3 / \alpha} \alpha^{-1} s^{3 / \alpha-1} d s d S(u) \\
= & \sum_{m=1}^{\infty}(2 \pi m \beta)^{-3 / 2} z^{m}(m \beta)^{-3 / \alpha} \alpha^{-1} \Gamma(3 / \alpha) \int_{\|u\|=1} V(u)^{-3 / \alpha} d S(u) \\
= & (2 \pi)^{-3 / 2}|\Lambda| \beta^{-3(2+\alpha) / 2 \alpha} \Gamma(1+3 / \alpha) g_{3(2+\alpha) / 2 \alpha}(z) \\
= & \varrho_{\beta . z}
\end{aligned}
$$

by Eqs. (2.7) and (2.10).

We now use this Lemma to prove the existence of the global density function for a general potential.

Theorem 2.6. Let $V$ be a general potential and let $0<z<1$. Then

$$
\varrho_{z}=\lim _{L \rightarrow \infty} \varrho_{L, z}
$$

exists and is given by

$$
\varrho_{z}=\int_{\mathbb{R}^{3}} \varrho_{z}(a) d^{3} a
$$

or explicitly

$$
\varrho_{z}=(2 \pi \beta)^{-3 / 2} \int_{\mathbb{R}^{3}} g_{3 / 2}\left(z e^{-\beta V(a)}\right) d^{3} a .
$$

Proof. We use the above Lemma together with the fact that a general potential dominates some homogeneous potential.

Given $0<z<1$ we choose $\gamma>0$ such that $z_{1}=z e^{\beta \gamma}<1$ and then using Equation 2.2 we choose $\varepsilon>0$ small enough so that

$$
V(x) \geqq-\gamma+\varepsilon\|x\|^{\delta}
$$

for all $x \in \mathbb{R}^{3}$. We let $\tilde{\varrho}_{L, z}$ denote the density function for the potential $\tilde{V}(x)=\varepsilon\|x\|$. We have the following inequalities, deduced immediately from the definitions.

$$
\begin{aligned}
& 0 \leqq \varrho_{L, z} \leqq \tilde{\varrho}_{L, z_{1}}, \\
& 0 \leqq \varrho_{L, z}(a) \leqq \tilde{\varrho}_{L, z_{1}}(a) .
\end{aligned}
$$

Now for each finite value of $L$

$$
\begin{aligned}
\int_{\mathbb{R}^{3}} \varrho_{L, z}(a) d^{3} a & =\int_{\mathbb{R}^{3}} \operatorname{tr}\left[\sigma_{L, z} N(\Omega+L a)\right] d^{3} a \\
& =L^{-3} \int_{\mathbb{R}^{3}} \operatorname{tr}\left[\sigma_{L, z} N(\Omega+x)\right] d^{3} x \\
& =\varrho_{L, z}
\end{aligned}
$$


by the countable additivity of the measure

$$
E \rightarrow \operatorname{tr}\left[\sigma_{L, z} N(E)\right]
$$

which we referred to earlier. Fatou's lemma immediately implies that

$$
\liminf _{L \rightarrow \infty} \varrho_{L, z} \geqq \int_{\mathbb{R}^{3}} \varrho_{z}(a) d^{3} a .
$$

The same argument applied to the family of non-negative integrable functions

yields

$$
\begin{gathered}
f_{L}(a)=\tilde{\varrho}_{L, z_{1}}(a)-\varrho_{L, z}(a) \\
\liminf _{L \rightarrow \infty}\left\{\tilde{\varrho}_{L, z_{1}}-\varrho_{L, z}\right\} \geqq \int_{\mathbb{R}^{3}}\left\{\tilde{\varrho}_{z_{1}}(a)-\varrho_{z}(a)\right\} d^{3} a .
\end{gathered}
$$

The result now follows upon application of Lemma 2.5 to the potential $\tilde{V}$.

We can now prove an almost complete generalisation of Lemma 2.3.

Theorem 2.7. Let $V$ be a general potential and let

$$
\varrho_{c}=(2 \pi \beta)^{-3 / 2} \int_{\mathbb{R}^{3}} g_{3 / 2}\left(e^{-\beta V(a)}\right) d^{3} a .
$$

Then if $0<\varrho<\varrho_{c}$ and $z(L)$ is defined by

$$
\varrho_{L, z(L)}=\varrho
$$

$z(L)$ converges to $z$ as $L \rightarrow \infty$ where

$$
\varrho=(2 \pi \beta)^{-3 / 2} \int_{\mathbb{R}^{3}} g_{3 / 2}\left(z e^{-\beta V(a)}\right) d^{3} a .
$$

On the other hand if $\varrho_{c} \leqq \varrho<\infty$ then $z(L)$ converges to 1 as $L \rightarrow \infty$.

The proof follows the same lines as for Lemma 2.3. We have not been able to obtain an estimate of the rate of convergence of $z(L)$ to 1 for the general potential, an unfortunate gap in the theory, which restricts the scope of some of our results below.

\section{§ 3. Expectation Functions of the Weyl Operators}

We recall some fundamental results on expectation functions of the Weyl operators in order to fix our notation [1]. The Weyl operator $W(f)$ is a unitary operator on $\mathscr{F}$ defined for all $f \in \mathscr{H}$ by

$$
W(f)=\exp \left[i\left\{a^{*}(f)+a(f)\right\} / \sqrt{2}\right]
$$

and satisfying the canonical commutation relations

$$
W(f) W(g)=W(f+g) \exp [i \operatorname{Im}\langle f, g\rangle / 2] .
$$


The expectation function of the Weyl operators is given by

where

$$
\operatorname{tr}\left[\sigma_{L, z} W(f)\right]=e^{-\|f\|^{2 / 4}} \exp \left[-\frac{1}{2} B_{L, z}(f, f)\right]
$$

$$
\begin{aligned}
B_{L, z}(f, f) & =\left\langle z e^{\beta E_{L, o}}\left(e^{\beta H_{L}}-z e^{\beta E_{L, 0}}\right)^{-1} f, f\right\rangle \\
& =\sum_{m=1}^{\infty} z^{m} e^{\beta m E_{L, 0}}\left\langle e^{-\beta m H_{L}} f, f\right\rangle \\
& =\sum_{n=0}^{\infty} \sum_{m=1}^{\infty} z^{m} e^{\beta m E_{L, 0}-\beta m E_{L, n}}\left|\left\langle f, \phi_{L, n}\right\rangle\right|^{2} .
\end{aligned}
$$

Since we are interested in the form of the field at distances from the origin which are very large compared with the thermal wavelength we try to calculate

$$
\lim _{L \rightarrow \infty} \operatorname{tr}\left[\sigma_{L, z} W\left(h_{L}\right)\right]
$$

where $h \in C_{0}^{\infty}\left(\mathbb{R}^{3}\right)$ and

$$
h_{L}(x)=h(x-L a)
$$

for some $a \in \mathbb{R}^{3}$. A similar idea to this has already been used in [13]. The problem usually considered $[1,12]$ corresponds to the case $a=0$.

Below the critical density for Bose-Einstein condensation the asymptotic form for the Weyl expectation functions may be calculated easily. Then

Theorem 3.1. Let $0<\varrho<\varrho_{c}$ and let $z(L)$ be the solution of $\varrho_{L, z(L)}=\varrho$.

$$
\begin{aligned}
\lim _{L \rightarrow \infty} B_{L, z(L)}\left(h_{L}, h_{L}\right) & =\sum_{m=1}^{\infty} z^{m} e^{-\beta m V(a)}\left\langle e^{-m \beta H_{0}} h, h\right\rangle \\
& =\int_{\mathbb{R}^{3}}\left\{z^{-1} e^{\beta V(a)+\beta\|x\|^{2} / 2}-1\right\}^{-1}|\hat{h}(x)|^{2} d^{3} x .
\end{aligned}
$$

Proof. We first take the special case $a=0$. If $z$ is chosen to satisfy Eq. (2.20), so that $0<z<1$ and if $z_{1}$ is chosen so that $z<z_{1}<1$ then

$$
0<e^{\beta E_{L, 0}} z(L) \leqq z_{1}
$$

for all large enough $L$. Therefore

$$
\begin{aligned}
\sum_{m=1}^{\infty} z(L)^{m} e^{\beta m E_{L, 0}}\left\langle e^{-\beta m H_{L}}\right. & h, h\rangle \\
& \leqq \sum_{m=1}^{\infty} z_{1}^{m}\|h\|^{2}<\infty .
\end{aligned}
$$

Therefore the series is dominated absolutely by a convergent series independent of $L$. Moreover by Equation 2.25 the individual terms of the series converge. This is enough to prove the equality of the first 
two ex pressions of the Theorem. For the last term we use Equation 2.26 to obtain a corresponding formula using Fourier transforms

$$
\left\langle e^{-t H_{0}} f, f\right\rangle=\int_{\mathbb{R}^{3}} e^{-t\|x\|^{2} / 2}|\hat{f}(x)|^{2} d^{3} x
$$

for all $f \in L^{2}\left(\mathbb{R}^{3}\right)$. The general case, where $a \neq 0$, can again be solved from the special case by considering the translated potential $V_{a}(x)=V(x+a)$.

Above the critical density for Bose-Einstein condensation the ground state makes a substantial contribution to the bilinear form $B$ in the thermodynamic limit. Following $[10,12]$ we therefore decompose $B$ into two components $S$ and $N$ corresponding to the superfluid and normal fluid respectively.

where

$$
B_{L, z}(f, f)=S_{L, z}(f, f)+N_{L, z}(f, f)
$$

and

$$
S_{L, z}(f, f)=z(1-z)^{-1}\left|\left\langle\phi_{L .0}, f\right\rangle\right|^{2}
$$

$$
\begin{aligned}
N_{L, z}(f, f) & =\sum_{n=1}^{\infty} \sum_{m=1}^{\infty} z^{m} e^{\beta m E_{L, 0}-\beta m E_{L, n}}\left|\left\langle\phi_{L, n}, f\right\rangle\right|^{2} \\
& =\sum_{m=1}^{\infty} z^{m} e^{\beta m E_{L, 0}}\left\{\left\langle e^{-\beta m H_{L}} f, f\right\rangle-e^{-\beta m E_{L, 0}}\left|\left\langle\phi_{L, 0}, f\right\rangle\right|^{2}\right\} .
\end{aligned}
$$

These two bilinear forms are then estimated separately.

As far as the normal fluid component is concerned we are only able to solve the problem completely for homogeneous potentials. This is because we need asymptotic estimates on $E_{L, 1}$ as $L \rightarrow \infty$ which seem difficult to obtain generally.

Theorem 3.2. Let $V$ be a homogeneous potential of order $\alpha$. Let $h \in C_{0}^{\infty}\left(\mathbb{R}^{3}\right)$ and let $h_{L}(x)=h(x-L a)$. Then

$$
\lim _{L \rightarrow \infty} N_{L . z}\left(h_{L}, h_{L}\right)=\sum_{m=1}^{\infty} z^{m} e^{-\beta m V(a)}\left\langle e^{-\beta m H_{0}} h, h\right\rangle
$$

uniformly in $z$ for $0 \leqq z \leqq 1$. If $\varrho>0$ and $z(L)$ is determined by $\varrho_{L, z(L)}=\varrho$ then

$$
\lim _{L \rightarrow \infty} N_{L . z(L)}\left(h_{L}, h_{L}\right)=\sum_{m=1}^{\infty} z^{m} e^{-\beta m V(a)}\left\langle e^{-\beta m H_{0}} h, h\right\rangle
$$

where $z$ is determined by Eq. (2.52) if $0<\varrho<\varrho_{c}$ and $z=1$ if $\varrho \geqq \varrho_{c}$; $\varrho_{c}$ is determined by Eq. (2.50).

Proof. For the usual reasons we need only treat the case $a=0$. We first show that the individual terms of the series of Eq. (3.9) converge to the individual terms of the series of Eq. (3.10). We have seen that $\left(H_{L}-E_{L, 0}\right)$ 
converges in the generalised sense to $\left(H_{0}+V(0)\right)$. If $f$ is the function on $\mathbb{R}$ defined by

$$
f(x)=\left\{\begin{array}{lll}
0 & \text { if } & x \leqq 0 \\
e^{-t x} & \text { if } & x>0
\end{array}\right.
$$

where $t>0$ then $f$ is bounded and continuous at every point except $x=0$, which is not in the point spectrum of $\left(H_{0}+V(0)\right)$; indeed this operator has no point spectrum. Therefore by [5]

$$
s-\lim _{L \rightarrow \infty} f\left(H_{L}-E_{L, 0}\right)=f\left(H_{0}+V(0)\right)
$$

and for any $h \in C_{0}^{\infty}\left(\mathbb{R}^{3}\right)$

$$
\begin{aligned}
\lim _{L \rightarrow \infty} e^{t E_{L, 0}}\left\{\left\langle e^{-t H_{L}} h, h\right\rangle-e^{-t E_{L, 0}}\left|\left\langle\phi_{L, 0}, h\right\rangle\right|^{2}\right\} \\
=\lim _{L \rightarrow \infty}\left\langle f\left(H_{L}-E_{L, 0}\right) h, h\right\rangle \\
=\left\langle f\left(H_{0}+V(0)\right) h, h\right\rangle \\
=e^{-t V(0)}\left\langle e^{-t H_{0}} h, h\right\rangle .
\end{aligned}
$$

To prove the first part of the theorem we now have to prove that the series is dominated absolutely by a convergent series independent of $z$ and $L$. Let $\Omega$ be a bounded region of volume $|\Omega|$ such that the support of $h$ is contained in $\Omega$ and let $P$ be the projection of $L^{2}\left(\mathbb{R}^{3}\right)$ onto $L^{2}(\Omega)$. Using the notation and results of [6], if $t>0$ then

$$
0 \ll P e^{-t H_{L}} \ll P e^{-t H_{0}}
$$

so

$$
\begin{aligned}
\left\|P e^{-t H_{L}}\right\| & \leqq\left\|P e^{-t H_{L}}\right\|_{\text {H.S. }} \\
& \leqq\left\|P e^{-t H_{0}}\right\|_{\text {H.S. }} \\
& =\left\{\operatorname{tr}\left[P e^{-2 t H_{0}}\right]\right\}^{1 / 2}=|\Omega|^{1 / 2}(4 \pi t)^{-3 / 4}
\end{aligned}
$$

by Equation 2.26. For arbitrary $0<\lambda<1 / 2$ we find by spectral theory that

$$
\begin{aligned}
& \mid\left\langle e^{-t H_{L}} h, h\right\rangle-e^{-t E_{L, 0}}\left\langle\left.\left\langle\phi_{L, 0}, h\right\rangle\right|^{2}\right| \\
& \quad \leqq\|h\|^{2}\left\|P\left(e^{-t H_{L}}-e^{-t E_{L, 0}} \phi_{L, 0} \otimes \phi_{L, 0}^{-}\right) P\right\| \\
& \quad=\|h\|^{2}\left\|\left(P e^{-\lambda t H_{L}}\right)\left(e^{-(1-2 \lambda) t H_{L}}-e^{-(1-2 \lambda) t E_{L, 0}} \phi_{L, 0} \otimes \phi_{L, 0}^{-}\right)\left(e^{-\lambda t H_{L}} P\right)\right\| \\
& \quad \leqq\|h\|^{2}|\Omega|(4 \pi t)^{-3 / 2} e^{-(1-2 \lambda) t E_{L, 1}} .
\end{aligned}
$$

Using Eq. (2.4) we choose $\lambda$ small enough so that

$$
(1-2 \lambda) E_{1,1}>E_{1,0}
$$


and observe from Eq. (1.15) that

$$
(1-2 \lambda) E_{L, 1}>E_{L, 0}
$$

for all $L$. Therefore

$$
\begin{aligned}
N_{L, z}(h, h) & =\sum_{m=1}^{\infty} z^{m} e^{\beta m E_{L, 0}}\left\{\left\langle e^{-\beta m H_{L}} h, h\right\rangle-e^{-\beta m E_{L, 0}}\left|\left\langle\phi_{L, 0}, h\right\rangle\right|^{2}\right\} \\
& \leqq \sum_{m=1}^{\infty} z^{m}\|h\|^{2}|\Omega|(4 \pi \beta m)^{-3 / 2} e^{\beta m E_{L, 0}-(1-2 \lambda) \beta m E_{L, 1}} \\
& \leqq \sum_{m=1}^{\infty}\|h\|^{2}|\Omega|(4 \pi \beta m)^{-3 / 2}<\infty
\end{aligned}
$$

This proves the first half of the Theorem. The second half is a consequence of the uniformity of the convergence and Theorem 2.7 .

We now turn to consideration of the bilinear form $S$. The basic result is

Proposition 3.3. If $0<\varrho<\varrho_{c}$ then

$$
\lim _{L \rightarrow \infty} S_{L, z(L)}\left(h_{L}, h_{L}\right)=0 .
$$

If $\varrho \geqq \varrho_{c}$ and

and

$$
\lim _{L \rightarrow \infty} L^{-3} z(L)\{1-z(L)\}^{-1}=k
$$

$$
\lim _{L \rightarrow \infty} L^{3 / 2} \phi_{L, 0}(L x)=\eta(x)
$$

locally uniformly on $\mathbb{R}^{3}$, then

$$
\lim _{L \rightarrow \infty} S_{L, z(L)}\left(h_{L}, h_{L}\right)=2 \pi k|\hat{h}(0)|^{2}|\eta(a)|^{2} .
$$

Proof. The first case is contained in the second for if $0<\varrho<\varrho_{c}$ then $z(L) \rightarrow z$ where $0<z<1$, and Eq. (3.21) is satisfied with $k=0$. Under the conditions of the Proposition

$$
\begin{aligned}
\lim _{L \rightarrow \infty} S_{L, z(L)}\left(h_{L}, h_{L}\right) \\
\quad=\lim _{L \rightarrow \infty} z(L)\{1-z(L)\}^{-1}\left|\left\langle\phi_{L, 0}, h_{L}\right\rangle\right|^{2} \\
\quad=\lim _{L \rightarrow \infty} z(L)\{1-z(L)\}^{-1}\left|\int_{\mathbb{R}^{3}} \phi_{L, 0}(x) \overline{h(x-L a)} d^{3} x\right|^{2} \\
\quad=\lim _{L \rightarrow \infty} L^{-3} z(L)\{1-z(L)\}^{-1}\left|\int_{\mathbb{R}^{3}} L^{3 / 2} \phi_{L, 0}\left\{L\left(L^{-1} x+a\right)\right\} \overline{h(x)} d^{3} x\right|^{2} \\
=k\left|\int_{\text {supp } h} \eta(a) \overline{h(x)} d^{3} x\right|^{2} \\
=2 \pi k|\eta(a)|^{2}|\hat{h}(0)|^{2} .
\end{aligned}
$$


Since we have proved the validity of Eq. (3.21), at least for homogeneous potentials [Eq. (2.21)], we turn now to the study of the asymptotic form of $\phi_{L, 0}$ as $L \rightarrow \infty$. In general this is a difficult problem and we content ourselves with some results for spherically symmetric potentials.

Theorem 3.4. Let $V$ be a spherically symmetric continuous potential such that $V(x)$ is a monotonically increasing function of $\|x\|$ with $V(x)=0$ if $0 \leqq\|x\| \leqq d, V(x)>0$ if $\|x\|>d$ and

Then if $d>0$

$$
\lim _{\|x\| \rightarrow \infty} V(x)=+\infty \text {. }
$$

$$
\lim _{L \rightarrow \infty} L^{3 / 2} \phi_{L, 0}(L x)=\eta(x)
$$

locally uniformly on $\mathbb{R}^{3}$ where

$$
\eta(x)= \begin{cases}(2 / d)^{1 / 2}\|x\|^{-1} \sin (\pi\|x\| / d) & \text { if }\|x\|<d \\ 0 & \text { if }\|x\| \geqq d .\end{cases}
$$

If $d=0$ then $\eta(x)=0$ if $x \neq 0$ with locally uniform convergence except at the origin and $\eta(0)=\infty$.

Proof. We observe that $\phi_{L, 0}$ is the unique solution in $L^{2}\left(\mathbb{R}^{3}\right)$ of

$$
H_{L} \phi=E_{L, 0} \phi
$$

so $\phi_{L .0}$ is rotationally invariant. Moreover by elliptic regularity theory $[19]$ it is continuous. If we define

$$
f_{L}(\|x\|)=\|x\| L^{3 / 2} \phi_{L, 0}(x)
$$

then $f_{L}$ is continuous on $[0, \infty), f_{L}(0)=0$ and $f_{L}$ is a weak, and hence strong, solution of the ordinary differential equation

$$
f_{L}^{\prime \prime}(r)=2 L^{2}\left(V(r)-E_{L, 0}\right) f_{L}(r) .
$$

Moreover $f_{L}$ is strictly non-negative on $(0, \infty)$ by $[20]$ and

$$
\int_{0}^{\infty}\left|f_{L}(r)\right|^{2} d r=1
$$

If $r_{L}$ is a solution of

$$
V\left(r_{L}\right)=E_{L, 0}
$$

then since $V$ is continuous $r_{L}>d$ and $r_{L} \rightarrow d$ as $L \rightarrow \infty$. The theory of ordinary differential equations [20] implies that $f_{L}$ is convex on $\left[0, r_{L}\right]$ and concave on $\left[r_{L}, \infty\right)$. Moreover $f_{L}^{\prime}(r)<0$ if $r \geqq r_{L}$ and $f_{L}(r) \rightarrow 0$ as $r \rightarrow \infty$ for each $L$. 
Now suppose $d>0$. In the interval $[0, d], f_{L}$ is given by

$$
f_{L}(r)=c_{L} \sin \left(\lambda_{L} r\right)
$$

where $c_{L}>0$ and $\lambda_{L}^{2}=2 E_{L, 0} L^{2}$. Since $f_{L}$ is strictly positive $\lambda_{L}<\pi / d$. We obtain a lower bound for $\lambda_{L}$ by comparison techniques. If $\varepsilon>0$ by explicit calculations there exist $d_{1}>d$ and $k>0$ such that the lowest eigenvalue of

$$
-\frac{d^{2}}{d r^{2}}+W(r)
$$

with the boundary condition $\psi(0)=0$ and

$$
W(r)=\left\{\begin{array}{lll}
0 & \text { if } & 0 \leqq r \leqq d_{1} \\
k & \text { if } & r>d_{1}
\end{array}\right.
$$

is greater than $\pi^{2} / d^{2}-\varepsilon$. But

$$
2 L^{2} V(r) \geqq W(r)
$$

for all large enough $L$, independent of $r$, so the minimax principle implies that for all large enough $L, \lambda_{L}^{2} \geqq \pi^{2} / d^{2}-\varepsilon$. Therefore

$$
\lim _{L \rightarrow \infty} \lambda_{L}=\pi / d \text {. }
$$

We next show that $c_{L}$ converges as $L \rightarrow \infty$. Let $s_{L}$ be the solution of

$$
V\left(s_{L}\right)=E_{L, 0}+n^{2} / 2 L^{2},
$$

where $n$ is arbitrary. Then $0<d<r_{L}<s_{L}$ and $s_{L} \rightarrow d$ as $L \rightarrow \infty$. If $0 \leqq r \leqq d$ then $f_{L}(r)=c_{L} \sin \left(\lambda_{L} r\right)$. If $d \leqq r \leqq s_{L}$ then $f_{L}(r) \leqq c_{L} \sin \left(\lambda_{L} d\right)$ since $f_{L}$ is monotonically decreasing for $r \geqq d$. If $r \geqq s_{L}$ then since $V$ is monotonically increasing

$$
2 L^{2}\left(V(r)-E_{L .0}\right) \geqq n^{2}
$$

which implies by [20, p. 165]

$$
f_{L}(r) \leqq c_{L} \sin \left(\lambda_{L} d\right) \exp \left[-n\left(r-s_{L}\right)\right] .
$$

These estimates together with Eq. (3.30) imply

$$
\lim _{L \rightarrow \infty} \mathcal{c}_{L}=(2 / d)^{1 / 2}
$$

so that $f_{L}$ converges in $L^{2}$-norm as well as locally uniformly to $\eta$.

The case $d=0$ is treated similarly.

For homogeneous spherically symmetric potentials we can combine the previous two results to obtain a complete description of the bilinear form $S$. 
Theorem 3.5. Let $V(x)=c\|x\|^{\alpha}$ and let $\varrho_{c}$ be defined by Eq. (2.19). If $\varrho \geqq \varrho_{c}$ let $z(L)$ be defined by Eq. 2.20. If $h \in C_{0}^{\infty}\left(\mathbb{R}^{3}\right)$ let $h_{L}(x)=h(x-L a)$. Then

$$
\lim _{L \rightarrow \infty} S_{L, z(L)}\left(h_{L}, h_{L}\right)=\left\{\begin{array}{rll}
0 & \text { if } & a \neq 0 \\
+\infty & \text { if } & a=0
\end{array} .\right.
$$

We comment on the results of this section. It has been widely believed that the ideal Boson gas has some pathological properties and that the introduction of interactions removes these. Our results enable us to make more precise the nature of these pathologies. Above the critical density $\varrho_{c}$ all the extra particles go into the ground state of the Hamiltonian, the phenomenon known as Bose-Einstein condensation [9]. The macroscopic spatial distribution of these particles is determined by the ground state wave function and in the limit $L \rightarrow \infty$ they concentrate exclusively at the origin. This is in sharp distinction to the normal fluid which we have seen in Lemma 2.5 is distributed with a density which varies throughout macroscopic space in an entirely regular manner. In another sense our conclusion fits in well with Lemma 2.5. We know that

$$
\varrho_{1}(a)=(2 \pi \beta)^{-3 / 2} g_{3 / 2}\left(e^{-\beta V(a)}\right)
$$

and for a homogeneous potential

$$
\varrho_{1}(a)<(2 \pi \beta)^{-3 / 2} g_{3 / 2}(1)
$$

for all $a \neq 0$. Therefore the normal fluid is only saturated at the origin, and this is the place where the superfluid is collected.

Nevertheless we have shown that for a homogeneous potential the spatial distributions of the normal fluid and superfluid are relatively singular in the limit $L \rightarrow \infty$, a conclusion which has also been found for the rotating Boson gas $[13,17]$. For non-homogeneous potentials the behaviour of the superfluid is in some ways even more peculiar, although the normal fluid behaves in a completely regular manner again. We refer to Theorem 3.4 where we showed that the superfluid is distributed in macroscopic space throughout the region

$$
\left\{x \in \mathbb{R}^{3}:\|x\| \leqq d\right\} .
$$

However within this class of potentials the constant $d$ does not vary continuously when the potential is perturbed by a small amount. That is the spatial distribution of the superfluid depends in an unstable manner on the potential.

It is perhaps worth mentioning here that for the ideal Boson gas there is a difference between the thermodynamic limits for the canonical ensemble and the grand canonical ensemble [10]. For an account of this see $[2,12]$. This difference is caused by the superfluid term and is again unstable under small perturbations [4]. 


\title{
References
}

1. Araki,H., Woods,E.J.: Representations of the canonical commutation relations describing a non-relativistic infinite free Bose gas. J. Math. Phys. 4, 637-662 (1963).

2. Cannon,J.T.: Infinite volume limits of the canonical free Bose gas states on the Weyl algebra. Commun. math. Phys. 29, 89--104 (1973).

3. Davies, E. B.: Quantum stochastic processes. Commun. math. Phys. 15, 277-304 (1969).

4. Davies, E. B.: The thermodynamic limit for an imperfect Boson gas. Commun. math. Phys. 28, 69-- 86 (1972).

5. Davies, E. B.: A generalisation of Kaplansky's theorem. J. London Math. Soc. 4, 435-436 (1972).

6. Davies, E. B.: Properties of the Green's functions of some Schrödinger operators. To appear, in J. London Math. Soc.

7. Garrod,C., Simmons, C.: Rigorous statistical mechanics for non-uniform systems. J. Math. Phys. 13, 1168-1176 (1972).

8. Gates, D. J., Penrose, O.: The van der Waals limit for classical systems. I. A variational principle. Commun. math. Phys. 15, 255-276 (1969).

9. Huang, K.: Statistical mechanics. London-New York: J. Wiley and Sons 1967.

10. Kac, M.: Private manuscript.

11. Kato, T.: Perturbation theory of linear operators. Berlin-Heidelberg-New York: Springer 1966.

12. Lewis, J.T., Pulè, J.: The equilibrium states of the free Boson gas. To appear.

13. Lewis, J.T., Pulè, J.: The free Boson gas in a rotating bucket. To appear.

14. Marchioro,C., Presutti,E.: Thermodynamics of particle systems in the presence of external macroscopic fields. I. Classical case. Commun. math. Phys. 27, 146-154 (1972).

15. Messiah, A.: Quantum mechanics Vol. 1. Amsterdam: North-Holland Publ. Co. 1965.

16. Millard, K.: A statistical mechanical approach to the problem of a fluid in an external field. J. Math. Phys. 13, 222-228 (1972).

17. Pulè, J.: D. Phil. thesis Oxford University 1972.

18. Ruelle, D.: Statistical mechanics, rigorous results. New York: W. A. Benjamin Inc. 1969.

19. Schechter, M.: Spectra of partial differential operators. Amsterdam North-Holland Publ. Co. 1971.

20. Titchmarsh,E.C.: Eigenfunction expansions, Part 1. Oxford Univ. Press, 2nd edition 1962.

21. Titchmarsh,E.C.: Eigenfunction expansions, Part 2. Oxford Univ. Press, 1958.

22. Watson, G. N.: Notes on generating functions of polynomials. J. London Math. Soc. 8, 194-199 (1933).

\author{
E. B. Davies \\ Mathematical Institute \\ Oxford University \\ 24-29 St. Giles \\ Oxford, U. K.
}


